\title{
Actividad antinflamatoria de extractos y fracciones obtenidas de cálices de Physalis peruviana L.
}

\author{
Luis A. Franco ${ }^{1}$, Germán E. Matiz ${ }^{1}$, Jairo Calle ${ }^{\dagger}$, Roberto Pinzón ${ }^{2}$, Luis F. Ospina ${ }^{2}$ \\ ${ }^{1}$ Departamento de Farmacia, Facultad de Ciencias Químicas y Farmacéuticas, Universidad de Cartagena, \\ Cartagena, Colombia. \\ 2 Departamento de Farmacia, Facultad de Ciencias, Universidad Nacional de Colombia, Bogotá, Colombia. \\ † Recientemente fallecido, Departamento de Farmacia, Facultad de Ciencias, Universidad Nacional de \\ Colombia, Bogotá D.C., Colombia.
}

Introducción. Los cálices de la uchuva, Physalis peruviana, son ampliamente utilizados en la medicina popular por sus propiedades como anticancerígeno, antimicobacterial, antipirético, diurético, immunomodulador y antinflamatorio.

Objetivo. Evaluar el efecto antinflamatorio de extractos y fracciones obtenidas de los cálices de Physalis peruviana en un modelo de inflamación aguda en ratón, procurando identificar las fracciones responsables de dicha actividad.

Materiales y métodos. Los cálices de Physalis peruviana fueron extraídos por percolación con solventes orgánicos. La fracción primaria hidroalcohólica se purificó mediante cromatografía en columna. La actividad antinflamatoria de los extractos y fracciones se evaluó utilizando el modelo murino de edema auricular inducido por 13-acetato de 12-tetradecanoilforbol.

Resultados. Se obtuvieron 38 fracciones secundarias en la cromatografía en columna de la fracción primaria hidroalcohólica, de las cuales seis fueron evaluadas en el modelo inflamatorio, mostrando actividad significativa $(p<0,05)$. La fracción mayoritaria identificada como $P p-D_{28}$ LF presentó una respuesta dosis dependiente con significativa inhibición del edema en dosis superiores a $250 \mu \mathrm{g} /$ oreja $(p<0,05)$.

Conclusión. Este estudio confirma la actividad antinflamatoria atribuida a los cálices de Physalis peruviana y también valida su uso en la medicina popular. Se identificaron las principales fracciones responsables de la actividad antinflamatoria, las cuales parecen ser promisorias para el desarrollo de fitopreparados. Se requieren estudios posteriores para aislar e identificar los compuestos responsables de la actividad y también para investigar el mecanismo involucrado en el efecto antinflamatorio observado.

Palabras clave: Solanaceae, Physalis, inflamación, agentes antinflamatorios, plantas medicinales.

Antiinflammatory activity of extracts and fractions obtained from Physalis peruviana L. calyces

Introduction. Cape gooseberry calyces (Physalis peruviana) have been used in folk medicine for their medicinal properties including anticancer, antimycobacterial, antipyretic, diuretic, immunomodulatory and antiinflammatory properties.

Objective. The antiinflammatory effect was evaluated for extracts and fractions obtained from Physalis peruviana calyces in a mice model of acute inflammation. The fractions responsible for antiinflammatory activity were extracted for possible identification.

Materials and Methods. The Physalis peruviana calyces were extracted by percolation with organic solvents. The primary hydroalcoholic fraction was purified by column chromatography. The antiinflammatory effect of extracts and fractions was evaluated using the 12-Otetradecanoylphorbol-13-acetate-induced mouse model of ear edema.

Results. Thirty-eight secondary fractions were obtained by column chromatography of primary hydroalcoholic fraction. Six fractions, evaluated in 12-O-tetradecanoylphorbol-13-acetateinduced inflammation assay, showed significant antiinflammatory activity $(p<0.05)$. The major 
fraction, $P p-D_{28}-L F$, showed a significant dose-dependent response at doses over $250 \mu \mathrm{g} / \mathrm{ear}$. Conclusion. The antiinflammatory activity attributed to Physalis peruviana calyces was confirmed and validated its use in folk medicine. Fractions responsible for the antiinflammatory action were identified and seem promising for phytomedicinal development. Further studies are needed to isolate and identify the active constituents of these fractions as well as to ascertain the mechanisms involved in the antiinflammatory effect.

Key words: Solanaceae, Physalis, inflammation, anti-inflammatory agents, plants, medicinal

Physalis es un importante género de la familia Solanaceae; cuenta con cerca de 120 especies que se distribuyen a través de las regiones tropicales y subtropicales del mundo, encontrándose principalmente en América, con un pequeño número de especies en Europa y en los países del suroriente y centro de Asia $(1,2)$. Physalis peruviana L., denominada comúnmente en Colombia como uchuva (Cape gooseberry), es una de las especies más conocidas de este género y es ampliamente utilizada en la medicina tradicional como anticancerígeno, antimicobacterial, antipirético, diurético, inmunomodulador y para el tratamiento de enfermedades como malaria, asma, hepatitis, dermatitis y artritis reumatoide (3). Los extractos de las hojas de esta planta han mostrado importantes actividades antibióticas, antioxidantes y antinflamatorias $(1,3,4)$. Los estudios fitoquímicos de este género han demostrado la presencia de witanólidos, esteroides, alcaloides, glicósidos y flavonoides (2,5-7).

Entre las muchas enfermedades que aquejan a la población mundial, las que involucran procesos inflamatorios representan un importante grupo; enfermedades como artritis reumatoidea, gota, asma o trastornos neurodegenerativos implican reacciones inflamatorias en algunos casos incapacitantes para quienes las padecen. Además, muchas otras dolencias menores que se presentan diariamente involucran procesos inflamatorios como respuesta natural del organismo ante traumas físicos y alergias. Como

\footnotetext{
Correspondencia:

Luis A. Franco Ospina, Departamento de Farmacia, Facultad de Ciencias Químicas y Farmacéuticas, Universidad de Cartagena, campus de Zaragocilla, Cartagena de Indias, Colombia. Tel: 57-5-6698179; Fax: 57-5-6698323

Ifrancoo@unicartagena.edu.co

Recibido: 21/09/06; aceptado: 057/02/07
}

consecuencia, existe una gran necesidad de descubrir y desarrollar nuevos agentes antinflamatorios más seguros y eficaces (8-10). El uso de las plantas medicinales o de sus componentes activos representa una alternativa cada vez más explorada y promisoria para el tratamiento de numerosos desórdenes inflamatorios $(11,12)$. Sin embargo, la limitada evidencia científica con respecto a la eficacia de estos derivados naturales, conjuntamente con la poca comprensión de los mecanismos de acción involucrados, ha limitado su incorporación a la práctica clínica (13).

Con este trabajo se pretende contribuir a la validación del uso popular antinflamatorio de los cálices de $P$. peruviana, evaluando bajo un esquema bioguíado el potencial antinflamatorio de sus extractos y fracciones por medio del modelo experimental agudo "edema auricular en ratón inducido por 13-acetato de 12-tetradecanoilforbol (TPA)", con el fin de identificar las fracciones responsables de la actividad antinflamatoria observada.

\section{Materiales y métodos}

\section{Material vegetal}

Los cálices de $P$. peruviana fueron recolectados en La Mesa, Cundinamarca, Colombia, en noviembre de 2003. La planta fue clasificada botánicamente por el Herbario Nacional Colombiano y una muestra quedó depositada en sus archivos como referencia (COL-512200).

\section{Fraccionamiento del material vegetal}

Los cálices secos y pulverizados de $P$. peruviana $(2 \mathrm{~kg})$ se sometieron a extracción por percolación con éter de petróleo (extracto etéreo total "A"); el marco residuo se extrajo posteriormente por el mismo método con etanol al $96 \%$ (extracto etanólico parcial "B"); el proceso en ambos casos se hizo hasta el agotamiento del material vegetal. 
Los extractos obtenidos en estos dos procesos se concentraron en rotavapor utilizando calentamiento suave $\left(30^{\circ} \mathrm{C}\right.$ a $\left.35^{\circ} \mathrm{C}\right)$ a presión reducida y se continuó su fraccionamiento bajo un esquema bioguíado utilizando el modelo de edema auricular. El extracto etéreo "A" $(271,6 \mathrm{~g})$ se disolvió en éter de petróleo y se fraccionó con una mezcla de $\mathrm{EtOH}-\mathrm{H}_{2} \mathrm{O}(9: 1)(3 \times 200 \mathrm{~mL})$. La fracción primaria hidroalcohólica resultante "D" $(223,3 \mathrm{~g})$ se fraccionó mediante cromatografía en columna con silicagel (Silicagel 60, Merck, 0,063-0,200 mm) eluyendo sucesivamente con éter de petróleo, diclorometano, acetato de etilo y etanol con verificación por cromatografía en capa delgada (CCD).

\section{Reactivos}

El 13-acetato de 12-tetradecanoilforbol (TPA) y la indometacina se obtuvieron de Sigma Chemical Co. (St. Louis, MO, USA); el éter de petróleo, el diclorometano, el acetato de etilo y el etanol, de Mallinckrodt Baker, Inc. (Phillipsburg, NY, USA), y los cromatofolios y la sílica gel, de Merck KGaA (Darmstadt, Alemania).

\section{Animales de experimentación}

Se utilizaron ratones ICR hembras de entre seis y siete semanas de edad, con pesos alrededor de $30 \mathrm{~g}$, suministrados por el Bioterio del Departamento de Farmacia de la Universidad Nacional de Colombia. Los experimentos se realizaron de acuerdo con los lineamientos del Comité de Ética de la Universidad Nacional de Colombia y a los lineamientos éticos internacionales para el cuidado y uso de animales de experimentación. Los animales se mantuvieron en un ambiente controlado (temperatura $22^{\circ} \mathrm{C} \pm 3^{\circ} \mathrm{C}$, humedad $70 \% \pm 5 \%$, ciclos de luz de 12 horas), con agua y alimento ad libitum. En el estudio se incluyó, además de las sustancias en estudio, un grupo control al cual sólo se le administró el vehículo y un grupo control positivo que recibió indometacina como fármaco de referencia.

\section{Edema auricular en ratón}

El edema auricular inducido por TPA se indujo de acuerdo al método descrito por De Young (14) y modificado por Payá (15). En éste, una solución de 13-acetato-12-O-tetradecanoilforbol $(2,5 \mu \mathrm{g})$ disuelto en acetona $(20 \mu \mathrm{L})$ se aplicó tópicamente sobre la superficie interna y externa de la oreja derecha del ratón (10 $\mu \mathrm{L} / \mathrm{lado})$ para provocar la inflamación. Las sustancias en estudio $(1 \mathrm{mg} /$ oreja) y la indometacina $(0,5 \mathrm{mg} /$ oreja) disueltas en acetona se aplicaron tópicamente sobre la superficie interna y externa de la oreja derecha del ratón inmediatamente antes de la administración del TPA. Trascurridas cuatro horas desde la administración del agente irritante, los animales se sacrificaron por dislocación cervical y se tomaron secciones circulares ( $7 \mathrm{~mm}$ de diámetro) de ambas orejas del animal (tratada y no tratada), las cuales se pesaron para determinar por diferencia el edema como delta de peso $\left(P_{\mathrm{t}}-P_{\mathrm{nt}}\right)$. Los resultados se expresan como este edema y como porcentaje de inhibición frente al grupo control, utilizando la siguiente expresión:

Porcentaje de inhibición $=\frac{\left(P_{t}-P_{n t}\right) \text { control }-\left(P_{t}-P_{n t}\right) \text { tratamientos }}{\left(P_{t}-P_{n t}\right) \text { control }} \times 100$

donde $P_{t}$ es el peso de la sección de la oreja tratada y $P_{n t}$ es el peso de la sección de la oreja no tratada (16).

\section{Análisis estadístico}

Los resultados se expresaron como la media \pm E.S.M. del delta de peso y se analizaron estadísticamente mediante análisis de varianza de una vía (ANOVA), seguido de un test de Dunnett para comparaciones múltiples. Los valores de $p<0,05$ se consideraron significativos.

\section{Resultados}

\section{Fraccionamiento del material vegetal}

Durante el proceso de extracción del material vegetal se obtuvieron dos extractos totales identificados como extracto etéreo total "A" $y$ extracto etanólico parcial " $\mathrm{B}$ " con rendimientos del $13,6 \%$ y $6,4 \%$, respectivamente. El fraccionamiento de $271,6 \mathrm{~g}$ del extracto etéreo total "A" condujo a la obtención de dos fracciones primarias identificadas como fracción primaria etérea "C" (13,8\% de rendimiento) y fracción primaria $\mathrm{EtOH}$ $\mathrm{H}_{2} \mathrm{O}$ “D” (82,2\% de rendimiento). El fraccionamiento en cromatografía en columna de 22 gramos de la fracción primaria $\mathrm{EtOH}-\mathrm{H}_{2} \mathrm{O}$ "D" condujo finalmente a la obtención de 38 fracciones secundarias, con 
seis fracciones principales que constituyen cerca del $90 \%$ del total fraccionado. La Pp- $D_{28}$-LF es la fracción mayoritaria con más del $37 \%$ (cuadro 1), evidenciándose en la verificación por CCD la presencia de tres compuestos.

\section{Edema auricular en ratón}

Los resultados de la evaluación de la actividad antinflamatoria del extracto total y de las fracciones primarias muestran que el extracto etéreo total "A" y la correspondiente fracción primaria $\mathrm{MeOH}$ $\mathrm{H}_{2} \mathrm{O}$ "D" presentaron significativa actividad antinflamatoria y, por lo tanto, fueron los que se fraccionaron bajo el esquema bioguíado (cuadro 2).

Las seis fracciones secundarias mayoritarias obtenidas de la fracción primaria " $D$ " se evaluaron en el modelo inflamatorio elegido. Como se observa en el cuadro 3, la administración tópica de estas fracciones reduce significativamente el edema auricular inducido por el TPA con porcentajes de inhibición superiores al $60 \%$ en todos los casos. La fracción mayoritaria $\mathrm{Pp}-\mathrm{D}_{28^{-}}$

Cuadro 1. Fracciones secundarias mayoritarias obtenidas de la columna cromatográfica de la fracción primaria hidroalcoholica "D".

\begin{tabular}{lrr}
\hline Fracción & Peso g $(\%)$ \\
\hline Pp- $D_{26}-$ LF & 0,99 & $(4,5)$ \\
Pp- $D_{27}-L F$ & 2,73 & $(12,4)$ \\
$P p-D_{28}-L F$ & 8,23 & $(37,4)$ \\
$P p-D_{29}-L F$ & 2,48 & $(11,3)$ \\
$P p-D_{30}-L F$ & 2,21 & $(10,0)$ \\
$P p-D_{31}-L F$ & 1,71 & $(7,8)$ \\
\hline
\end{tabular}

Cuadro 2. Actividad antinflamatoria del extracto total y fracciones primarias obtenidas de cálices de Physalis peruviana.

\begin{tabular}{lcc}
\hline Grupos & $\begin{array}{c}\text { Dosis } \\
\text { (mg/oreja) }\end{array}$ & $\begin{array}{c}\text { Porcentaje } \\
\text { de } \\
\text { inhibición }\end{array}$ \\
\hline Indometacina & 0,5 & $64,7^{*}$ \\
Extracto etéreo total (A) & 1,0 & $56,2^{*}$ \\
Extracto etanólico parcial (B) & 1,0 & 6,8 \\
Fracción primaria etérea (C) & 1,0 & 3,4 \\
Fracción primaria MeOH- ${ }_{2} \mathrm{O}$ (D) & 1,0 & $67,2^{*}$ \\
\hline
\end{tabular}

Los resultados representan la media E.S.M. $(n=10)$

$\left({ }^{*} p<0,05\right.$ ANOVA, estadísticamente significativo frente al control).
LF mostró una potente actividad antinflamatoria con una inhibición cercana al $70 \%$, comparable a la presentada por el fármaco de referencia (indometacina).

En función de los importantes resultados obtenidos para la fracción mayoritaria $\mathrm{Pp}-\mathrm{D}_{28}$ - $\mathrm{LF}$, se realizó un estudio dosis respuesta de su actividad en el modelo de edema auricular inducido por TPA, empleando dosis de 1.000, 500, 250 y $100 \mu \mathrm{g} /$ oreja. Los resultados presentados en la figura 1 muestran un efecto dosis dependiente de esta fracción con una $\mathrm{DE}_{50} 454 \mu \mathrm{g} / \mathrm{oreja}$ (límites de confianza del $95 \%$ entre 277 y $757 \mu \mathrm{g} /$ oreja). Se

Cuadro 3. Actividad antinflamatoria de las fracciones secundarias mayoritarias obtenidas de la fracción primaria hidroalcoholica "D".

\begin{tabular}{|c|c|c|c|}
\hline Grupos & $\begin{array}{c}\text { Dosis } \\
\text { (mg/oreja) }\end{array}$ & $\begin{array}{c}\text { Edema } \\
(\mathrm{mg})\end{array}$ & \% Inhibición \\
\hline Control & ---- & $19,7 \pm 0,7$ & \\
\hline Indometacina & 0,5 & $5,8 \pm 0,8$ & $70,3^{*}$ \\
\hline$P p-D_{26}-L F$ & 1,0 & $3,2 \pm 0,3$ & $83,9^{*}$ \\
\hline$P p-D_{27}^{26}-L F$ & 1,0 & $6,5 \pm 0,4$ & $67,0^{*}$ \\
\hline $\mathrm{Pp}-\mathrm{D}_{28}-\mathrm{LF}$ & 1,0 & $5,3 \pm 0,5$ & $73,2^{*}$ \\
\hline $\mathrm{Pp}-\mathrm{D}_{29}-\mathrm{LF}$ & 1,0 & $6,7 \pm 0,6$ & $66,0^{*}$ \\
\hline$P p-D_{30}-L F$ & 1,0 & $7,6 \pm 0,7$ & $61,6^{*}$ \\
\hline$P p-D_{31}-L F$ & 1,0 & $7,8 \pm 0,7$ & $60,2^{*}$ \\
\hline
\end{tabular}

Los resultados representan la media EE.S.M. $(n=10)$ ( ${ }^{*} p<0,05$ ANOVA, estadísticamente significativo frente al control).

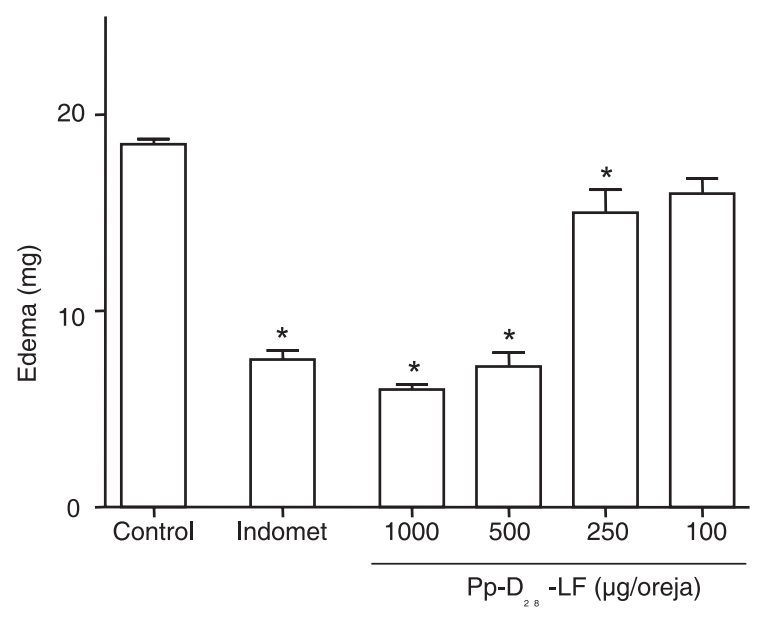

Figura 1. Efecto dosis respuesta de la fracción secundaria $\mathrm{Pp}-\mathrm{D}_{28}$-LF de Physalis peruviana sobre el edema auricular inducido por TPA. ${ }^{*} P<0,05$ ANOVA, estadísticamente significativo frente al control. 
observa una significativa y comparable reducción del edema inducido por el TPA para las dosis de 1.000 y $500 \mu \mathrm{g} /$ oreja: $6,0 \pm 0,2$ y $7,2 \pm 0,7 \mathrm{mg}$, respectivamente $(p<0,05$ frente a $18,5 \pm 0,3 \mathrm{mg}$ en el grupo control). Con dosis más bajas se pierde gradualmente la actividad de esta fracción, de forma que la dosis de $100 \mu \mathrm{g} /$ oreja no muestra actividad.

\section{Discusión}

Se evaluó la actividad antinflamatoria de extractos totales y fracciones primarias y secundarias obtenidas de los cálices de $P$. peruviana con un método extensamente utilizado para evaluar sustancias antinflamatorias como es el edema auricular inducido por TPA. El proceso inflamatorio desencadenado por la aplicación tópica del TPA se debe a la activación de la proteína cinasa $C$ (PKC) dérmica, la cual inicia la respuesta inflamatoria. Todos los agentes antinflamatorios muestran actividad en este modelo, pero principalmente los inhibidores duales COX/LOX $(16,17)$, aunque los inhibidores de la cicloxigenasa (COX) parecen ser más efectivos que los inhibidores de la lipoxigenasa (LOX) para reducir la respuesta edematosa (18); en este orden de ideas, se puede pensar que las fracciones de $P$. peruviana pueden tener un comportamiento similar a los inhibidores de la COX en este modelo.

El fraccionamiento del extracto etéreo total " $A$ " produjo en cada paso fracciones con creciente actividad antinflamatoria, indicando un enriquecimiento del activo como consecuencia de la purificación. Se obtuvieron seis fracciones secundarias que representan cerca del $90 \%$ del total fraccionado, las cuales presentan significativa actividad antinflamatoria, de tal forma que el efecto antinflamatorio observado en los cálices de $P$. peruviana se debe principalmente a la acción aditiva de los diferentes constituyentes de estas fracciones.

La fracción $P p-D_{28}-L F$ se consideró como la principal responsable de la actividad antinflamatoria observada en los cálices de $P$. peruviana, ya que constituye la fracción mayoritaria de la fracción primaria "D", obteniéndose en el fraccionamiento realizado 8,2 gramos, que representan poco más del $37 \%$ del total fraccionado. Además, mostró importantes propiedades antinflamatorias, inhibiendo significativamente de manera dosis dependiente el edema auricular agudo inducido por TPA, con una inhibición comparable a la presentada por la indometacina en la misma dosis ( $500 \mu \mathrm{g} /$ oreja). Los estudios posteriores realizados con esta fracción demostraron que se encuentra constituida en un $82 \%$ por la mezcla de dos ésteres de sacarosa no reportados en la naturaleza (manuscrito en preparación).

Este es uno de los pocos estudios realizados sobre los cálices de $P$. peruviana y uno de los primeros en lo que respecta al estudio de su actividad antinflamatoria. Estos resultados, conjuntamente con los obtenidos por Choi et al. (10) en extractos de Physalis angulata, constituyen unos de los primeros reportes que muestran actividad antinflamatoria in vivo de extractos del género Physalis.

Los resultados de este estudio validan el uso de los cálices de $P$. peruviana en la medicina popular para el tratamiento de enfermedades inflamatorias como el reumatismo. Además, se logró identificar las principales fracciones responsables de la actividad antinflamatoria, las cuales se obtienen fácilmente y con excelentes rendimientos, hecho que las hace muy promisorias para el efectivo desarrollo de preparados fitoterapéuticos. Estos resultados son prometedores e invitan a continuar estudios sobre estas fracciones a fin de aislar sus compuestos activos y evaluarlos en otros modelos de inflamación tanto agudos como crónicos, así como de determinar los posibles mecanismos implicados en el efecto farmacológico mediante su evaluación frente a mediadores específicos del proceso inflamatorio como las prostaglandinas, el óxido nítrico, la mieloperoxidasa y el factor de necrosis tumoral, además de examinar su capacidad de actuar como captadoras de radicales libres.

\section{Agradecimientos}

Los autores agradecen a Ricardo Gaitán, Claudio Gómez y al profesional en química Helver Lesmes por sus útiles aportes en el desarrollo de este documento; igualmente al Departamento de Farmacia de la Facultad de Ciencias de la Universidad Nacional, a la Facultad de Ciencias 
Químicas y Farmacéuticas de la Universidad de Cartagena y a Colciencias por su valioso apoyo.

\section{Conflicto de Intereses}

Los autores manifiestan que no tienen ningún conflicto de intereses respecto a la realización y difusión de este trabajo.

\section{Financiación}

Este trabajo fue financiado por Colciencias, código 1101-05-11454, la Universidad Nacional de Colombia y la Universidad de Cartagena.

\section{Referencias}

1. Ahmad S, Malik A, Yasmin R, Ullah N, Gul W, Khan PM et al. Whitanolides from Physalis peruviana. Phytochemistry 1999;50:647-51

2. Bastos GN, Santos AR, Ferrerira VM, Costa AM, Bispo Cl, Silveira AJ et al. Antinociceptive effect of the aqueous extract obtained from roots of Physalis angulata L. on mice. J Ethnopharmacol 2006;103:241-5.

3. Wu SJ, Ng LT, Huang YM, Lin DL, Wang SS, Huang SN et al. Antioxidant activities of Physalis peruviana. Biol Pharm Bull 2005;28:963-6.

4. Wu SJ, Tsai JY, Chang SP, Lin DL, Wang SS, Huang SN et al. Supercritical carbon dioxide extract exhibits enhance antioxidant and anti-inflammatory activities of Physalis peruviana. J Ethnopharmacol 2006;108:407-13.

5. Mayorga $\mathbf{H}$, Duque C, Knapp $\mathbf{H}$, Winterhalter $\mathbf{P}$. Hydroxyester disaccharides from fruits of cape gooseberry (Physalis peruviana). Phytochemistry 2002;59:439-45.

6. Pérez-Castorena AL, García M, Martínez M, Maldonado E. Physalins from Physalis solanaceus. Biochem Syst Ecol 2004;32:1231-4.

7. Wu SJ, Ng LT, Lin DL, Huang SN, Wang SS, Lin CC. Physalis peruviana extract induces in human Hep G2 cells through CD95/CD95L system and the mitochondrial signaling transduction pathway. Cancer Lett 2004;215:199-208.

8. Yesilada E, Üstun O, Sezik E, Takaishi Y, Ono Y, Honda G. Inhibitory effects of Turkish folk remedies on inflammatory cytokine: interleukin- $1 \alpha$, interleukin- $1 \beta$ and tumour necrosis factor $\alpha$. J Ethnopharmacol 1997;58:59-73.
9. Srinivasan K, Muruganandan S, Lal J, Chandra S, Tandan SK, Prakash VR. Evaluation of antiinflammatory activity of Pongamia pinnata leaves in rats. J Ethnopharmacol 2001;78:151-7.

10. Choi EM, Hwang J. Investigations of anti-inflammatory and antinociceptive activities of Piper cubeba, Physalis angulata and Rosa hybrida. J Ethnopharmacol 2003;89:171-5

11. Sautebin L. Prostaglandins and nitric oxide as molecular targets for anti-inflammatory therapy. Fitoterapia 2000;71(Suppl. 1):S48-57.

12. Ukil A, Maity S, Karmakar S, Datta N, Vedasiromoni JR, Das PK. Curcumin, the major component of food flavour turmeric, reduces mucosal injury in trinitrobenzene sulphonic acid-induced colitis. $\mathrm{Br} \mathrm{J}$ Pharmacol 2003;139:209-18.

13. Maity S, Ukil A, Karmakar S, Datta N, Chaudhuri T, Vedasiromoni JR et al. Thearubigin, the major polyphenol of black tea, ameliorates mucosal injury in trinitrobenzene sulfonic acid-induced colitis. Eur J Pharmacol 2003;470:103-12.

14. De Young LM, Kheifets JB, Ballaron SJ, Young JM. Edema and cell infiltration in the phorbol ester-treated mouse ear are temporally separated and can be differentially modulated by pharmacologic agents. Agents Actions 1989;26:335-41.

15. Payá M, Ferrándiz ML, Sanz MJ, Bustos G, Blasco R, Ríos JL et al. Study of the antioedema activity of some seaweed and sponge extracts from the mediterranean coast in mice. Phytother Res 1993;7:159-62.

16. Jiménez-Estrada M, Chilpa RR, Apan TR, Lledias F, Hansberg W, Arrieta D et al. Anti-inflammatory activity of cacalol and cacalone sesquiterpenes isolated from Psacalium decompositum. J Ethnopharmacol 2006;105:34-8.

17. Recio MC, Cérda-Nicolas M, Potterat $O$, Hamburger M, Rios JL. Anti-inflammatory and antiallergic activity in vivo of lipophilic Isatis tinctoria extracts and Tryptanthrin. Planta Med 2006;72:539-46.

18. García MD, Fernandez M, Alvarez A, Saenz M. Antinociceptive and anti-inflammatory effect of the aqueous extract from leaves of Pimenta racemosa var. ozua (Mirtaceae). J Ethnopharmacol 2004;91:69-73. 